\title{
La incorporación de las TIC en las empresas. Factores de la brecha digital en las Mipymes de Aguascalientes*
}

\author{
The Incorporation of ICT in Firms. Factors of the Digital \\ Divide in MSMEs of Aguascalientes
}

\author{
Héctor Edgar Buenrostro Mercado ${ }^{* *}$ y María del Carmen Hernández Eguiarte ${ }^{* * *}$
}

\begin{abstract}
RESUMEN
La incorporación de las TIC en las empresas, es considerada como un elemento que aumenta su competitividad a través de una mayor productividad, eficiencia y rentabilidad de la inversión. Pero su efecto depende de las características propias de la tecnología, de las condiciones del entorno, así como de las capacidades internas de las firmas. En el presente trabajo el objetivo es identificar cuáles son los factores internos de las Mipymes que inciden en la incorporación de las TIC dentro de sus actividades de producción, a partir de las etapas de propuestas por la ONU y Cepal, por medio de un modelo Probit, realizado con los datos de una encuesta aplicada a 1,006 empresas del estado de Aguascalientes. Los resultados muestran que las diferencias en la incorporación de las TIC se deben fundamentalmente a los conocimientos y capacidades de los trabajadores y directivos de las Mipymes y al equipamiento especializado, que se refleja en mayor flexibilidad para la incorporación de cambios en la organización.
\end{abstract}

Palabras clave: TIC, brecha digital, Mipymes, modelos Probit, Aguascalientes.

Clasificación JEL: C25 O33 O14.

\begin{abstract}
The incorporation of ICT in companies is considered as an element that increases their competitiveness through greater productivity, efficiency and profitability of investment. But its effect depends on the characteristics of technology, the environmental conditions, as well as the internal capacities of firms. In the present paper, the objective is to identify what are the internal factors of MSMEs that affect the incorporation of ICT within their production activities, from the proposals stages by the UN and ECLAC, starting from a Probit model, made with data from a survey applied to 1,006 companies in the state of Aguascalientes. The results show that the differences in the incorporation of ICT are mainly due to the knowledge and skills of the workers and managers of MSMEs and specialized equipment, which is reflected in greater flexibility for the incorporation of changes in the organization.
\end{abstract}

Keywords: ICT, digital divide, MSMEs, Probit model, Aguascalientes. JEL Classification: C25 O33 O14.

\footnotetext{
* Fecha de recepción: 13/06/2017. Fecha de aceptación: 23/05/2018.

** Centro de Investigación e Innovación en Tecnologías de la Información y Comunicación, México. E-mail: hector. buenrostro@infotec.mx. ORCID:0000-0003-0527-8864.

*** Investigadora independiente, México. E-mail: meguiarte@gmail.com. ORCID:0000-0002-4310-2504.
} 


\section{INTRODUCCIÓN}

La incorporación de las TIC en las empresas ha impulsado el crecimiento de la economía en los últimos años (Rivas y Stumpo, 2013). Trabajos empíricos realizados en diferentes países muestran un efecto positivo (Wielicki y Arendt, 2010; Consoli, 2012), no sólo en los aspectos relacionados con una mayor intensidad del capital y el aumento de la automatización que se refleja en términos de la productividad, eficiencia, cuota de mercado y rentabilidad, sino en aquellos vinculados indirectamente al uso de la tecnología, que se refieren a los cambios organizacionales, así como a los procesos y usos de la información y comunicación, y el desarrollo de nuevas modalidades de acceso al conocimiento (Bayo-Moriones y Lera-López, 2007; Cepal, 2013; Santinha y Soares, 2004).

A partir de la década de los ochenta, toma impulso el estudio de la incorporación de las TIC en las empresas a través de la correlación entre su uso y la productividad, considerando que se integraban de manera lineal y se distribuían de forma homogénea dentro de las organizaciones (Molina, Rotondo y Yoguel, 2013). Uno de los principales acercamientos teóricos surge a finales de esta década desde los estudios de la información: el Modelo de Aceptación Tecnológica (TAM), que, a través de las percepciones de facilidad de uso y utilidad, intenta explicar la difusión de las tecnologías de la información (Davis, 1989), que ha sido retomado y enriquecido posteriormente por una gran cantidad de autores (Gefen y Straub, 2000).

Desde la teoría económica, a partir de la década de los noventa surge una extensa literatura, donde por medio de un enfoque micro, se presentan diferencias de adopción entre las empresas debido a factores individuales, organizacionales, tecnológicos, económicos y del entorno (Consoli, 2012; Dewan y Riggins, 2005). Muestran que las Mipymes se encuentran en desventaja respecto a las grandes empresas en el uso de TIC (Dewan y Riggins, 2005), ya que están sujetas a limitantes económicos, técnicos y humanos para incorporarse de manera eficiente a la tecnología (Consoli, 2012). Por lo que en los últimos años ha sido un tema de creciente interés el identificar los elementos particulares que inciden en la determinación de la adopción de la tecnología, con el fin de incentivar su uso de manera más eficiente dentro de los procesos de producción para aumentar la competitividad de estas firmas.

El presente trabajo tiene como objetivo identificar cuáles son los factores internos de las Mipymes que inciden en la incorporación de las TIC dentro de sus actividades de producción y administración. Para ello se hace uso de una encuesta realizada en 2014 a algunas empresas del estado de Aguascalientes, usando la clasificación de intensidad de uso propuesta por la OCDE y Cepal, basada en cuatro etapas que agrupan la apropiación de las tecnologías de la información 
en las actividades internas de las firmas (OCDE-Cepal, 2012; Kotelnikov, 2007), para determinar cuáles factores son relevantes en cada una de ellas.

Aun cuando a nivel internacional hay diversas investigaciones en relación con la brecha digital en las empresas (Consoli, 2012), este es uno de los pocos trabajos que aborda los determinantes del uso de las TIC en los procesos productivos de las Mipymes en un estado territorial de México, ya que no se cuenta con ejercicios sistemáticos a nivel nacional que aporten información detallada al respecto.

El documento se divide en cinco apartados; el primero expone una discusión sobre la relevancia de las TIC y los trabajos que han abordado los factores determinantes en su adopción por parte de las Mipymes. En el segundo se presenta la metodología y el modelo en la investigación. En el tercero se exponen los resultados del análisis estadístico. En el cuarto se presenta el análisis y discusión de los resultados para finalmente presentar las conclusiones del trabajo.

\section{LA BRECHA DIGITAL EN LAS MIPYMES}

Las Mipymes ${ }^{1}$ desarrollan un papel central en la economía por su aporte al crecimiento económico, el empleo y a la generación de innovaciones. Además, representan más del 95 por ciento de las empresas de los países en desarrollo (Ueki, Tsuji y Cárcamo, 2005). Por lo que es fundamental comprender la manera cómo estos agentes incorporan a las TIC dentro de los procesos de creación de valor, a través de un manejo más eficiente de sus recursos tecnológicos.

La incorporación de las TIC cobra mayor relevancia en la medida en que la formación de ventajas competitivas, fundamentadas en la generación y acumulación de capacidades tecnológicas, se convierte en la base del crecimiento económico (OCDE-Cepal, 2012), su uso incentiva cambios en las actividades de las firmas, así como entre socios, clientes y proveedores, modificando los procesos de producción y distribución (Galve y Gargallo, 2004; Wielicki y Arendt, 2010; Hawash y Lang, 2010).

\footnotetext{
En el presente trabajo, se considera lo establecido por el Dof del 30 de junio de 2009, donde se definen a las Mipymes industriales como aquellas que cuentan con un máximo de 250 empleados y a las Mipymes de servicios y de comercio como aquellas que cuentan con hasta 100 empleados (DOF, 30 de junio 2009).
} 


\section{I.1. La evolución del concepto de brecha digital}

Los primeros trabajos sobre brecha digital se orientaban al análisis de la cantidad y el tipo de empresas que contaban con acceso a la tecnología en relación con aquellas que no lo tenían. De manera fundamental, se concentraban en la determinación de la infraestructura, la disponibilidad de las computadoras y la accesibilidad a las TIC (Cruz-Jesus, Oliveira y Bacao, 2012; Pejic, Zorojaa y Bosilj, 2013).

En trabajos posteriores, el concepto de brecha digital se modificó cambiando hacia un fenómeno multifacético que incorpora las capacidades de utilizar apropiadamente las TIC (Arent, 2008; Dewan y Riggins, 2005; Tello-Leal, 2008; Tello-Leal, Sosa, Reyna y Tello-Leal, 2012), resultando una concepción de dos dimensiones; por un lado, la infraestructura y su adopción (llamada de primer orden) y por otro, la desigualdad en la capacidad de utilizar las TIC entre los usuarios que cuentan con acceso a esta tecnología (señalada como de segundo orden), las cuales son abordadas a nivel individual, de organización y nacional (Cruz-Jesus et al., 2012; Pejic, Zorojaa y Bosilj, 2013; Arent, 2008).

Los estudios de la adopción de las TIC en las empresas se han orientado en los últimos años al análisis de la brecha digital de segundo orden. En particular, se han encauzado hacia los modelos de los procesos de difusión de la tecnología y el grado de intensidad de uso entre los que se encuentran:

1. Los modelos epidémicos, que asumen que la difusión de la tecnología se realiza por medio del contacto con firmas que ya cuentan con la tecnología;

2. los modelos Probit, que presentan la probabilidad de que una empresa acceda a la tecnología a partir de la información con que cuenta;

3. los modelos de stock, que sostienen que las firmas harán uso de las TIC siempre y cuando se refleje en una reducción de costos mayor a sus competidores y,

4. los modelos de orden, que asumen que las empresas que adoptan primero la tecnología obtienen mayores beneficios que aquellas que lo hacen posteriormente (Galliano y Roux, 2008; Forman y Goldfarb, 2005).

Estos acercamientos se han orientado, en primer lugar, a considerar los impactos de las TIC como el estudio de los costos y beneficios, en donde su incorporación está generando cambios en las actividades de las empresas, así como entre socios, clientes y proveedores (Galve y Gargallo, 2004; Wielicki y Arendt, 2010; Novick, Rotondo y Yoguel, 2013; Hawash y Lang, 2010). En segundo lugar, en los elementos del medio ambiente y de la organización que influyen en la adopción de la tecnología. En particular, aquellos que se encuentran en relación con la capacidad tecnológica del país, el perfil productivo, la capacidad tecno-organizacional, la estructura de las articulaciones entre las empresas, la 
vinculación con instituciones de educación superior y centros de investigación, así como las capacidades internas, la estructura organizacional, la posición en la cadena productiva y la experiencia en el uso de las TIC (Pejic, Zorojaa y Bosilj, 2013; Bazini, Ilia y Qarri, 2011; Arent, 2008; Consoli, 2012).

\section{I.2. La determinación de la brecha digital}

En la concepción de la brecha digital de primero y segundo orden, se generaron un conjunto de escalas para determinar el grado de incorporación de la tecnología dentro de los procesos de las empresas. En el caso del presente trabajo se utilizó el modelo propuesto por la ONU y Cepal, que consta de cuatro etapas de intensidad de uso de las TIC.

1. Etapa 1. Son aquellas empresas que no cuentan con acceso a las TIC para realizar sus actividades diarias, en particular no hacen uso de computadoras y/o no tienen servicio de Internet.

2. Etapa 2. Son empresas que cuentan con acceso a las TIC básicas, que requieren de una inversión reducida, cuyo equipamiento e infraestructura se restringe hacia el uso de computadoras y el acceso a Internet. Las herramientas informáticas que se emplean no demandan un conocimiento especializado, por ejemplo: suites ofimáticas, correo electrónico, servicios bancarios y del sector público. El empleo de la tecnología se concentra en tareas de gestión y administración de carácter rutinario, que inciden en la estandarización y agilización de los procesos, por lo que su impacto en la productividad es limitado, debido al empleo poco sofisticado de los equipos.

3. Etapa 3. En estas empresas, las TIC posibilitan la modificación de los procesos de información para la toma de decisiones y la articulación de las áreas estratégicas, incorporándose a lo largo del proceso productivo e involucrando a los proveedores con las distintas áreas de la firma. El uso de estas herramientas informáticas requiere de la formación de habilidades generadas a través de la capacitación de los empleados y de la estandarización de los procesos de administración, modificando la organización para adaptarla a la incorporación de la tecnología.

4. Etapa 4. En esta etapa se encuentran las empresas que hacen un uso intensivo y complejo de las TIC, que se expresa en el uso de intranet en combinación con software especializado tales como ERP (Enterprise Resource Planning), CRM 
(Customer Relationship Management), BPM (Business Process Management), CMS (Content Management System) y BI (Business Intelligence). Requiere de recursos humanos altamente calificados y de una infraestructura tecnológica que dé soporte a las áreas internas (OCDE-Cepal, 2012; Kotelnikov, 2007).

A medida que se avanza en las etapas, se incorporan usos más complejos de las TIC que requieren de mayores capacidades de los trabajadores, así como una creciente integración entre los procesos productivos y administrativos. De manera paralela, el uso de la tecnología fortalece los canales para la articulación con los agentes externos, facilitando el acceso a las cadenas globales de producción (Rovira, Santoleri y Stumpo, 2013; Arendt, 2008; Hollenstein, 2004).

\section{I.3. Factores de adopción de las TIC en las Mipymes}

Debido a su heterogeneidad, las Mipymes presentan diferentes grados de incorporación de la tecnología, que dependen de una combinación de dimensiones internas y externas que inciden en su implementación:

1. Las características propias de la tecnología de acuerdo con su nivel de desarrollo;

2. los elementos propios de la organización que inciden en el uso de las TIC en las empresas, generando procesos de adopción lentos y complejos que forman barreras a la adopción de esta tecnología, y

3. los factores derivados del entorno que, en el caso de los países en desarrollo, son fundamentales para explicar su limitada adopción por parte de las Mipymes (Arendt, 2008; Cepal-UE, 2010).

Si bien la infraestructura de acceso, las políticas y programas de apoyo y los avances en la tecnología son precursores clave para la incorporación de las TIC en las empresas, no garantizan beneficios por sí mismos, ya que los elementos propios de las organizaciones juegan un papel central, a través de impulsar el crecimiento por medio del uso de las TIC de manera efectiva dentro de los procesos de producción y gestión, para agregar valor a los productos (Matthews, 2007; Consoli, 2012). Se observa que la disponibilidad de hardware y software y el acceso a la conectividad no se traduce en mejoras evidentes en los procesos de negocio (Arendt, 2008).

En investigaciones realizadas en diferentes países y regiones se identifican elementos internos y externos a las empresas que inciden en la formación de la brecha digital (Pejic, Zorojaa y Bosilj, 2013; Pick y Nishida, 2015). En relación 
con los factores internos, se han generado diferentes enfoques para analizar los obstáculos que desalientan el uso de las TIC en las Mipymes.

En el presente trabajo se consideraron las condiciones internas que inciden en su incorporación, las cuales se dividieron en cuatro categorías para su análisis: $i$ ) las características de las empresas, $i$ i) las capacidades de los empleados, iii) el equipamiento y $i v$ ) las condiciones administrativas internas (Bayo-Moriones y Lera-López, 2007; Consoli, 2012; Bazini, Ilia y Qarri, 2011; Fabiani, Schivardi y Trento, 2005; Forman y Goldfarb, 2005; Ifinedo, 2012; Wielicki y Cavalcanti, 2006; Arendt, 2008), las que se muestran en la figura 1.

Figura 1. Factores internos de las empresas que inciden en la adopción de TIC.

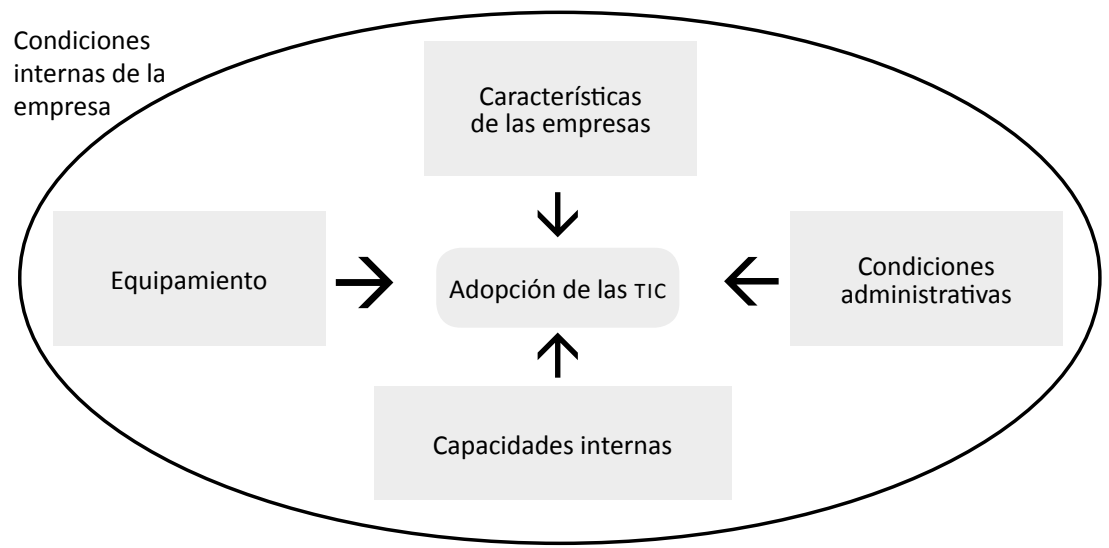

Fuente: elaboración propia con base en Bayo-Moriones y Lera-López, 2007; Wielicki y Cavalcanti, 2006; Arendt, 2008.

Dentro de las características de las empresas se consideran elementos propios de las organizaciones tales como el tamaño, antigüedad y sector, que inciden en el gasto asociado a los equipos, el software, el acceso a las redes, el mantenimiento y soporte técnico (Pejic, Zorojaa y Bosilj, 2013; Bayo-Moriones y Lera-López, 2007; Arendt, 2008; Bazini, Ilia y Qarri, 2011; Fabiani, Schivardi y Trento, 2005; Forman y Goldfarb, 2005).

El equipamiento agrupa al equipo necesario, tanto software como hardware general y especializado, para las actividades de las empresas, que forman un elemento que favorece la disminución de la brecha digital (Bazini, Ilia y Qarri, 2011; Consoli, 2012). Por otro lado, los conocimientos y habilidades del responsable de la administración son fundamentales para la reducción de la brecha 
digital, debido a que es quien se encarga de la toma de decisiones respecto a la incorporación de las TIC en la empresa (Consoli, 2012; Arendt, 2008; Bayo-Moriones y Lera-López (2007).

Finalmente, de trabajos anteriores se desprende que las Mipymes presentan deficiencias en las capacidades internas en TIC, lo que hace necesario que los responsables de las empresas inviertan en la formación de los trabajadores para impulsar las habilidades básicas para facilitar la aplicación de estas herramientas en la producción y administración. (Arendt, 2008; Pejic, Zorojaa y Bosilj, 2013; Bayo-Moriones y Lera-López, 2007; Bazini, Ilia y Qarri, 2011; Dewan y Riggins, 2005; Fabiani, Schivardi y Trento, 2005).

\section{I.4. Los determinantes del uso de las TIC en las Mipymes de México}

Con la generalización del uso de las computadoras, son pocas las empresas que no las han incorporado en el desarrollo de sus actividades diarias (OCDE-Cepal, 2012), pero la información de uso de las TIC en México es muy escasa y se circunscribe a los indicadores relacionados con la infraestructura y equipamiento, sin considerar elementos más complejos relacionados con su incorporación en la gestión y producción de los diferentes sectores económicos.

El estudio de la brecha digital de segundo orden en México ha sido un tema recurrente desde la mitad de la década del siglo XX. Algunos buscaban establecer los diferenciales de uso a través de las condiciones de acceso asociadas a condiciones económicas, sociales y políticas (Mariscal, 2005), determinando los elementos que inciden en las disparidades de penetración de las TIC en la población en general, en diferentes regiones del país. Otros trabajos se enfocan en grupos sociales específicos como herramienta para explicar las diferencias entre el uso y su papel como elemento de inclusión social y económica (Tello-Leal, SosaReyna y Tello-Leal, 2012).

En el caso particular de las empresas, los trabajos apuntan a problemas relacionados con la falta de recursos humanos con conocimientos en herramientas especializadas en la producción, limitaciones presupuestarias para la atracción y retención de personal calificado y una falta de implementación de cambios en la organización y gestión de las Mipymes (Tello-Leal, 2008; Cepal, 2013), como resultado de la falta de planeación en la incorporación de las TIC, que se refleja en limitadas mejoras en los flujos de información y una baja estandarización de procesos, ineficiencias en la gestión y producción y falta de descentralización de las decisiones restringiendo el potencial aporte de la tecnología en la producción (Novick, Rotondo y Yoguel, 2013; Billón, Lera y Ortiz, 2007; Schatan y Enríquez, 2014). 
El análisis del estado de Aguascalientes es relevante por su elevado grado de industrialización, cuya tradición se remonta al siglo XIX y que se consolida en la década de los ochenta, con el fortalecimiento de los sectores automotriz, textil y de servicios (Casalet, et al., 2008). Así, en el 2014 el número de empresas en el estado asciende a 55,464 con una participación de 1.12 por ciento del PIB nacional (Inegi, 2015). Con la expansión de las telecomunicaciones inalámbricas, la cobertura de servicios como el teléfono y el Internet, ha presentado una rápida adopción de las TIC entre las Mipymes del estado (García y Martínez, 2009), por lo que es un caso que permite explorar las diferencias que se presentan entre ellas, en un entorno con facilidades de acceso a la tecnología.

Las condiciones de Aguascalientes (altamente industrializado y con elevado nivel de infraestructura de telecomunicaciones) han generado interés por analizar los niveles de incorporación de las TIC en el sector productivo. Los trabajos existentes se insertan dentro de investigaciones que analizan las condiciones de las empresas locales para mejorar sus condiciones y niveles de competitividad a través de las TIC (García y Martínez, 2009; Martínez, García y Maldonado, 2010; Maldonado, Martínez, García, Aguilera y González, 2010) y se orientan en la determinación de los niveles de innovación, gestión del conocimiento y cultura empresarial.

Como resultado del análisis de trabajos anteriores, en relación con los determinantes de la adopción de las TIC en las empresas, se elaboró la siguiente hipótesis: dentro de los determinantes internos que inciden en la incorporación de las TIC en las Mipymes de Aguascalientes, los más importantes son aquellos que se refieren a las capacidades internas y en menor medida a los costos de acceso a los equipos tecnológicos (equipamiento), la ausencia de adaptación de los procesos al uso de la tecnología (condiciones administrativas) y a las actividades propias del sector económico al que pertenece la empresa (características de la empresa).

A continuación, se describe el modelo utilizado para contrastar la hipótesis, así como los resultados obtenidos para su posterior análisis, en función del modelo de adopción propuesto por la ONU y Cepal. 


\section{METODOLOGÍA}

Por las características de los datos con que se cuentan, los cuales son un corte transversal en el tiempo, y que sólo pueden tomar dos valores 0 y 1 (uso o falta de uso de las TIC en las actividades de la empresa), se propuso la aplicación de un modelo probabilístico (Probit) para poner a prueba la hipótesis sobre los determinantes que inciden en la adopción de las TIC en las Mipymes (Gujarati y Porter, 2010).

Los datos utilizados en la investigación se obtuvieron a través de una encuesta a un conjunto de 1,200 unidades económicas agrupadas en siete sectores económicos por clase de empresa, seleccionadas de acuerdo con el Directorio Estadístico Nacional de Unidades Económicas (DENUE) del estado de Aguascalientes, bajo el método de muestreo aleatorio simple. El tamaño de la muestra fue calculado con la finalidad de obtener resultados con un nivel de confianza del 95 por ciento, teniendo un índice de respuesta de 83.3 por ciento de los encuestados (1,006 empresas).

El levantamiento de los datos se realizó en dos etapas, la primera en el mes de febrero del 2012, correspondiente a una prueba piloto para ajustar el instrumento y la segunda en abril del 2014, donde se aplicó un cuestionario compuesto por 35 preguntas cerradas y de opción múltiple, distribuidas en seis bloques: i) identificación de la empresa; ii) equipamiento tecnológico; iii) uso de software; iv) recursos y capacitación; $v$ ) uso de herramientas de internet, y vi) retos y oportunidades en el uso de las TIC.

A partir de la base de datos, se ubicó a las empresas encuestadas en alguna de las cuatro categorías propuestas por OCDE-Cepal, cuyas variables se encuentran en el Anexo 1. La razón de su clasificación fue que al menos contara con el 50 por ciento de las características propias de cada etapa. De este tratamiento se obtuvieron cuatro vectores dicotómicos, donde 1 indica la pertenencia a la etapa correspondiente y 0 cualquier otro caso.

Los vectores de las etapas se definieron como variables dependientes del modelo probabilístico que se calculó a través del software Stata V.13, para explicar el comportamiento de la variable dependiente binaria mediante la implementación de una función de distribución acumulativa normal. La distribución logística normal estándar de esta clase de modelos se caracteriza por tener media cero y varianza igual a 1 (Gujarati y Porter, 2010; Greene, 1998).

Las variables explicativas fueron concentradas en cuatro vectores de acuerdo con las características internas de la organización presentadas en la figura 1:i) condiciones de la firma, ii) condiciones administrativas de la firma, iii) equipamiento de la firma, y iv) capacidades de la firma. El conjunto de variables que compone cada uno de los vectores, así como su decodificación se muestra en el cuadro 1. 
Cuadro 1. Variables explicativas de los modelos Probit.

\begin{tabular}{|c|c|c|c|}
\hline Vector & Variable & Descripción & Codificación \\
\hline \multirow{15}{*}{$\begin{array}{l}\text { Características } \\
\text { de las } \\
\text { empresas }\end{array}$} & \multirow{3}{*}{ Tamaño } & \multirow{3}{*}{ Tamaño de la empresa } & 1 Microempresa \\
\hline & & & 2 Pequeña empresa \\
\hline & & & 3 Mediana empresa \\
\hline & Antemp & Antigüedad de la empresa & Antigüedad en años \\
\hline & \multirow{6}{*}{ Sector } & \multirow{6}{*}{ Sector al que pertenece la empresa } & Construcción \\
\hline & & & Manufactura \\
\hline & & & Terciario, comercio y transporte \\
\hline & & & Servicios varios \\
\hline & & & Educación y salud \\
\hline & & & $\begin{array}{l}\text { Esparcimiento, hoteles } \\
\text { y restaurantes }\end{array}$ \\
\hline & \multirow{5}{*}{ presupuestoc } & \multirow{5}{*}{$\begin{array}{l}\text { Porcentaje del presupuesto destina- } \\
\text { do a sistemas o equipo de cómputo }\end{array}$} & 00 por ciento \\
\hline & & & 11 por ciento- 2 por ciento \\
\hline & & & 22 por ciento- 5 por ciento \\
\hline & & & 35 por ciento-10 por ciento \\
\hline & & & 410 por ciento o más \\
\hline \multirow{5}{*}{$\begin{array}{l}\text { Condiciones } \\
\text { administrativas } \\
\text { de las } \\
\text { empresas }\end{array}$} & \multirow[b]{2}{*}{ Ctrfam } & \multirow{2}{*}{$\begin{array}{l}\text { Control mayoritario de la empresa } \\
\text { (si el grupo familiar tiene más del } \\
50 \text { por ciento del capital y el gerente } \\
\text { es familiar) }\end{array}$} & 1 Sí \\
\hline & & & 0 No \\
\hline & \multirow{2}{*}{ Grefam } & \multirow{2}{*}{$\begin{array}{l}\text { Puestos de la dirección ocupados } \\
\text { mayoritariamente por miembros } \\
\text { de la familia }\end{array}$} & $1 \mathrm{Si}$ \\
\hline & & & 0 No \\
\hline & Antdir & $\begin{array}{l}\text { Antigüedad del director general/ } \\
\text { gerente de la empresa }\end{array}$ & Antigüedad en años \\
\hline \multirow{7}{*}{$\begin{array}{l}\text { Condiciones } \\
\text { administrativas } \\
\text { de las } \\
\text { empresas }\end{array}$} & \multirow{7}{*}{ años_esco } & \multirow{7}{*}{$\begin{array}{l}\text { Años de escolaridad del director } \\
\text { general/gerente de la empresa }\end{array}$} & 09 o menos años \\
\hline & & & $1 \mathrm{Al}$ menos 10 años \\
\hline & & & $2 \mathrm{Al}$ menos 13 años \\
\hline & & & $3 \mathrm{Al}$ menos 14 años \\
\hline & & & $4 \mathrm{Al}$ menos 17 años \\
\hline & & & $5 \mathrm{Al}$ menos 20 \\
\hline & & & $6 \mathrm{Al}$ menos 22 años \\
\hline
\end{tabular}


ECONOMÍA TEORÍA Y PRÁCTICA [ISSN: 2448-7481] • Nueva Época, año 27, número 50, enero-junio 2019 Héctor Edgar Buenrostro Mercado y María del Carmen Hernández

Cuadro 1. Continuación.

\begin{tabular}{|c|c|c|c|}
\hline Vector & Variable & Descripción & Codificación \\
\hline \multirow{14}{*}{$\begin{array}{l}\text { Equipamiento } \\
\text { de la empresa }\end{array}$} & Cantdce & $\begin{array}{l}\text { Número de computadoras de } \\
\text { escritorio que dispone la empresa }\end{array}$ & Unidades \\
\hline & Cantport & $\begin{array}{l}\text { Número de computadoras portátiles } \\
\text { que dispone la empresa }\end{array}$ & Unidades \\
\hline & Cantserv & $\begin{array}{l}\text { Número de servidores que dispone } \\
\text { la empresa }\end{array}$ & Unidades \\
\hline & Cantmov & $\begin{array}{l}\text { Número de dispositivos móviles } \\
\text { que dispone la empresa }\end{array}$ & Unidades \\
\hline & Canotros & $\begin{array}{l}\text { Número de otros dispositivos } \\
\text { que dispone la empresa }\end{array}$ & Unidades \\
\hline & \multirow{3}{*}{ Equipred } & \multirow{3}{*}{$\begin{array}{l}\text { Los equipos de cómputo de la } \\
\text { empresa están en red }\end{array}$} & 1 Sí \\
\hline & & & $0 \mathrm{No}$ \\
\hline & & & 9 No aplica \\
\hline & \multirow{2}{*}{ lic_libre } & \multirow{2}{*}{$\begin{array}{l}\text { La empresa cuenta con al menos } \\
\text { un programa con licencia libre }\end{array}$} & 1 Sí \\
\hline & & & O No \\
\hline & \multirow{2}{*}{ lic_comercial } & \multirow{2}{*}{$\begin{array}{l}\text { La empresa cuenta con al menos } \\
\text { un programa con licencia comercial }\end{array}$} & 1 Sí \\
\hline & & & $0 \mathrm{No}$ \\
\hline & \multirow{2}{*}{ lic_propia } & \multirow{2}{*}{$\begin{array}{l}\text { La empresa utiliza al menos un } \\
\text { programa con licencia propia }\end{array}$} & 1 Sí \\
\hline & & & O No \\
\hline \multirow{12}{*}{$\begin{array}{l}\text { Capacidades } \\
\text { de la empresa }\end{array}$} & Antuso & $\begin{array}{l}\text { Antigüedad en el uso de equipo } \\
\text { de cómputo }\end{array}$ & Antigüedad en años \\
\hline & \multirow{3}{*}{ Personalcap } & \multirow{3}{*}{$\begin{array}{l}\text { Personal de la empresa que utiliza } \\
\text { equipos y programas de cómputo } \\
\text { está capacitado }\end{array}$} & 1 Sí \\
\hline & & & $0 \mathrm{No}$ \\
\hline & & & 9 No aplica \\
\hline & Persoinf & $\begin{array}{l}\text { Personal ocupado en funciones } \\
\text { de informática }\end{array}$ & Personas \\
\hline & \multirow{3}{*}{ Progcap } & \multirow{3}{*}{$\begin{array}{l}\text { Capacitación del personal en } \\
\text { el manejo de tecnologías de } \\
\text { información y comunicaciones }\end{array}$} & 1 Sí \\
\hline & & & 0 No \\
\hline & & & 9 No aplica \\
\hline & Cursosanual & $\begin{array}{l}\text { Cursos en promedio que se } \\
\text { ofrecen al año }\end{array}$ & Número de cursos \\
\hline & \multirow{3}{*}{ Unidsi } & \multirow{3}{*}{$\begin{array}{l}\text { La empresa cuenta con una unidad } \\
\text { de sistemas de informática }\end{array}$} & 1 Sí \\
\hline & & & $0 \mathrm{No}$ \\
\hline & & & 9 No aplica \\
\hline
\end{tabular}

Fuente: elaboración propia. 
Por lo tanto, la especificación funcional de los modelos Probit aquí aplicados es la siguiente:

Donde:

$$
\operatorname{Etapa} 1(2,3,4)=\beta_{0}+\beta_{i 1} C F+\beta_{i 2} R F+\beta_{i 3} E F+\beta_{i 4} K H+U_{i}
$$

Etapa $1(2,3,4)=$ Vector de pertenencia a la etapa 1, 2, 3 o 4

$\mathrm{CF}=$ Vector de variables de condiciones de la firma

$\mathrm{RF}=$ Vector de variables de condiciones administrativas de la firma

$\mathrm{EF}=$ Vector de variables de equipamiento de la firma

$\mathrm{KH}=$ Vector de variables de capacidades de la firma

$\mathrm{U}_{\mathrm{i}}=$ Término de error

Sea $P_{i}$ igual a uno la probabilidad de que una Mipyme pertenezca a una etapa dado el conjunto de variables explicativas, entonces en caso contrario, cuando sea cero, la probabilidad será de $1-P_{i}$, lo que se expresa de la siguiente forma:

$$
E\left(Y_{i} \mid X_{i}\right)=0\left(1-P_{i}\right)+1\left(P_{i}\right)=P_{i}
$$

Donde $Y_{i}$ se refiere al evento de pertenencia de cada una de las etapas y $X_{i}$ el conjunto de variables explicativas.

\section{RESULTADOS}

En el cuadro siguiente se muestran los resultados del modelo Probit con los datos de la encuesta a las Mipymes de Aguascalientes. En las columnas se encuentran las variables dependientes, representadas por cada una de las etapas de uso de las TIC de acuerdo con la clasificación de la OCDE-Cepal, a las cuales les corresponde el valor dicotómico 0 y 1 . En las filas de la 2 a la 29 se presentan los vectores de los factores internos de las empresas que inciden en la adopción de TIC (figura 1).

En cada celda se encuentra el efecto marginal de las variables explicativas para cada etapa de intensidad de uso de TIC definidas anteriormente, incluyendo la significancia estadística a niveles de error de 1,5 y 10 por ciento. 
ECONOMÍA TEORÍA Y PRÁCTICA [ISSN: 2448-7481] • Nueva Época, año 27, número 50, enero-junio 2019 Héctor Edgar Buenrostro Mercado y María del Carmen Hernández

\section{Cuadro 2. Resultados del modelo Probit.}

\begin{tabular}{|c|c|c|c|c|c|c|c|c|}
\hline \multicolumn{4}{|c|}{ Etapas } & (1) & Etapa 1 & Etapa 2 & Etapa 3 & Etapa 4 \\
\hline \multirow{9}{*}{$\begin{array}{l}\text { Caracterís- } \\
\text { ticas de las } \\
\text { empresas }\end{array}$} & \multicolumn{2}{|c|}{ Tamaño de la empresa } & Tamano & $(2)$ & $\begin{array}{r}0.0299943 \\
* * * \\
\end{array}$ & $\begin{array}{r}-0.1012133 \\
* * *\end{array}$ & 0.0001455 & $\begin{array}{r}0.022822 \\
* * *\end{array}$ \\
\hline & \multicolumn{2}{|c|}{ Antigüedad de la empresa } & Antemp & (3) & n.a. & -0.0005976 & 0.0020724 & -0.0006012 \\
\hline & \multirow{6}{*}{$\begin{array}{l}\text { Sector al } \\
\text { que per- } \\
\text { tenece la } \\
\text { empresa }\end{array}$} & Construcción & Construccion & (4) & $\begin{array}{r}-0.0352635 \\
* *\end{array}$ & -0.0420931 & 0.115412 & 0.011728 \\
\hline & & $\begin{array}{l}\text { Industria } \\
\text { manufacturera }\end{array}$ & Manufactura & $(5)$ & -0.0200427 & -0.0166894 & 0.0914312 & -0.0098325 \\
\hline & & $\begin{array}{l}\text { Terciario, } \\
\text { comercio y } \\
\text { transporte }\end{array}$ & Comytransp & $(6)$ & -0.0082695 & -0.0751673 & $\begin{array}{r}0.1330664 \\
* *\end{array}$ & -0.0004167 \\
\hline & & $\begin{array}{l}\text { Servicios } \\
\text { varios }\end{array}$ & Servarios & (7) & -0.0004936 & $\begin{array}{r}0.1205647 \\
*\end{array}$ & $\begin{array}{r}-0.1330224 \\
*\end{array}$ & 0.0113348 \\
\hline & & $\begin{array}{l}\text { Educación } \\
\text { y salud }\end{array}$ & Eduysalud & (8) & -0.0078225 & $\begin{array}{r}0.2094024 \\
*\end{array}$ & $\begin{array}{r}-0.1269631 \\
*\end{array}$ & 0.003921 \\
\hline & & $\begin{array}{l}\text { Esparcimien- } \\
\text { to, hoteles y } \\
\text { restaurantes }\end{array}$ & $\begin{array}{l}\text { Esparci- } \\
\text { miento }\end{array}$ & (9) & 0.0074554 & 0.0607952 & -0.0668218 & 0.0114099 \\
\hline & $\begin{array}{l}\text { Porcentaje } \\
\text { destinado } \\
\text { equipo de }\end{array}$ & $\begin{array}{l}\text { el presupuesto } \\
\text { sistemas o } \\
\text { mputo }\end{array}$ & $\begin{array}{l}\text { presupues- } \\
\text { toc }\end{array}$ & (10) & n.a. & 0.000011 & n.a. & n.a. \\
\hline \multirow{4}{*}{$\begin{array}{l}\text { Condiciones } \\
\text { administra- } \\
\text { tivas de las } \\
\text { empresas }\end{array}$} & \multicolumn{2}{|c|}{$\begin{array}{l}\text { Control mayoritario de la } \\
\text { empresa (si el grupo familiar } \\
\text { tiene más del } 50 \text { por ciento } \\
\text { del capital y el gerente es } \\
\text { familiar) }\end{array}$} & Ctrfam & (11) & -0.017943 & 0.0596648 & -0.0456956 & 0.0265239 \\
\hline & \multicolumn{2}{|c|}{$\begin{array}{l}\text { Puestos de la dirección } \\
\text { ocupados mayoritariamente } \\
\text { por miembros de la familia }\end{array}$} & Grefam & $(12)$ & 0.0012907 & -0.0304264 & 0.027204 & -0.0352164 \\
\hline & \multicolumn{2}{|c|}{$\begin{array}{l}\text { Antigüedad del director ge- } \\
\text { neral/gerente de la empresa }\end{array}$} & Antdir & (13) & n.a. & $\begin{array}{r}0.0035882 \\
*\end{array}$ & -0.0001283 & 0.0001995 \\
\hline & \multicolumn{2}{|c|}{$\begin{array}{l}\text { Años de escolaridad del } \\
\text { director general/gerente } \\
\text { de la empresa }\end{array}$} & años_esco & (14) & $\begin{array}{r}-0.0025199 \\
*\end{array}$ & -0.0018144 & 0.0049939 & 0.0016715 \\
\hline \multirow{5}{*}{$\begin{array}{l}\text { Equipa- } \\
\text { miento de la } \\
\text { empresa }\end{array}$} & \multicolumn{2}{|c|}{$\begin{array}{l}\text { Número de computadoras } \\
\text { de escritorio que dispone } \\
\text { la empresa }\end{array}$} & CantDCE & (15) & $\begin{array}{r}-0.0029385 \\
*\end{array}$ & -0.0030209 & $\begin{array}{r}0.0034189 \\
*\end{array}$ & -0.0003511 \\
\hline & \multicolumn{2}{|c|}{$\begin{array}{l}\text { Número de computadoras } \\
\text { portátiles que dispone la } \\
\text { empresa }\end{array}$} & Cantport & (16) & $\begin{array}{r}-0.0255976 \\
* * *\end{array}$ & -0.0024526 & -0.0059403 & 0.0013092 \\
\hline & \multicolumn{2}{|c|}{$\begin{array}{l}\text { Número de servidores que } \\
\text { dispone la empresa }\end{array}$} & Cantserv & (17) & $\begin{array}{r}-0.0150774 \\
*\end{array}$ & $\begin{array}{r}-0.0542868 \\
* * *\end{array}$ & -0.0012191 & $\begin{array}{r}0.0070417 \\
* *\end{array}$ \\
\hline & \multicolumn{2}{|c|}{$\begin{array}{l}\text { Número de dispositivos } \\
\text { móviles que dispone la } \\
\text { empresa }\end{array}$} & Cantmov & (18) & 0.0038155 & 0.0073525 & -0.0011158 & -0.0001624 \\
\hline & \multicolumn{2}{|c|}{$\begin{array}{l}\text { Número de otros dispositi- } \\
\text { vos que dispone la empresa }\end{array}$} & Canotros & (19) & n.a. & -0.0299789 & $\begin{array}{r}0.2040372 \\
*\end{array}$ & n.a. \\
\hline
\end{tabular}


Cuadro 2. Continuación.

\begin{tabular}{|c|c|c|c|c|c|c|c|}
\hline & Etapas & & (1) & Etapa 1 & Etapa 2 & Etapa 3 & Etapa 4 \\
\hline \multirow{4}{*}{$\begin{array}{l}\text { Equipa- } \\
\text { miento de la } \\
\text { empresa }\end{array}$} & $\begin{array}{l}\text { Los equipos de cómputo } \\
\text { de la empresa están en red }\end{array}$ & Equipred & (20) & $\begin{array}{r}-0.0657619 \\
* * *\end{array}$ & -0.0210934 & $\begin{array}{r}0.227713 \\
* * *\end{array}$ & 0.0230896 \\
\hline & $\begin{array}{l}\text { La empresa cuenta con al } \\
\text { menos un programa con } \\
\text { licencia libre } \\
\end{array}$ & lic_libre & (21) & $\begin{array}{r}-0.0544919 \\
* * *\end{array}$ & 0.0128537 & 0.0594614 & 0.0346535 \\
\hline & $\begin{array}{l}\text { La empresa cuenta con al } \\
\text { menos un programa con } \\
\text { licencia comercial }\end{array}$ & lic_comercial & (22) & $\begin{array}{r}-0.1569406 \\
* * *\end{array}$ & -0.075359 & $\begin{array}{r}0.240862 \\
* * *\end{array}$ & $\begin{array}{r}0.0205742 \\
*\end{array}$ \\
\hline & $\begin{array}{l}\text { La empresa utiliza al menos } \\
\text { un programa con licencia } \\
\text { propia }\end{array}$ & lic_propia & (23) & $\begin{array}{r}-0.0671953 \\
* * *\end{array}$ & $\begin{array}{r}-0.1015834 \\
* * *\end{array}$ & $\begin{array}{r}0.2048914 \\
* * * \\
\end{array}$ & 0.0093169 \\
\hline \multirow{6}{*}{$\begin{array}{l}\text { Capacida- } \\
\text { des de la } \\
\text { empresa }\end{array}$} & $\begin{array}{l}\text { Antigüedad en el uso de } \\
\text { equipo de cómputo }\end{array}$ & Antuso & (24) & n.a. & $\begin{array}{r}-0.0094075 \\
* * *\end{array}$ & n.a. & 0.000912 \\
\hline & $\begin{array}{l}\text { Personal de la empresa que } \\
\text { utiliza equipos y programas } \\
\text { de cómputo está capacitado }\end{array}$ & Personalcap & (25) & n.a. & $\begin{array}{r}-0.057941 \\
*\end{array}$ & n.a. & $\begin{array}{r}0.0342992 \\
* *\end{array}$ \\
\hline & $\begin{array}{l}\text { Personal ocupado en funcio- } \\
\text { nes de informática }\end{array}$ & PersolNF & (26) & n.a. & n.a. & n.a. & 0.001994 \\
\hline & $\begin{array}{l}\text { Capacitación del personal } \\
\text { en el manejo de TIC }\end{array}$ & Progcap & (27) & n.a. & $\begin{array}{r}-0.0832745 \\
* *\end{array}$ & n.a. & -0.0138564 \\
\hline & $\begin{array}{l}\text { Cursos en promedio que se } \\
\text { ofrecen al año }\end{array}$ & Cursosanual & (28) & n.a. & -0.0021004 & n.a. & -0.001035 \\
\hline & $\begin{array}{l}\text { La empresa cuenta con una } \\
\text { unidad de sistemas } \\
\text { de informática }\end{array}$ & UnidSI & (29) & n.a. & -0.0407681 & n.a. & $\begin{array}{r}0.0559677 \\
* * *\end{array}$ \\
\hline \multicolumn{2}{|c|}{ Pseudo R2 de Mc Fadden } & & (30) & 0.4401 & 0.1991 & 0.1365 & 0.2939 \\
\hline \multicolumn{2}{|l|}{$P(Y i=1 \mid X)$} & & (31) & 0.0418 & 0.1807 & 0.5497 & 0.0219 \\
\hline
\end{tabular}

Fuente: elaboración propia.

Significancia estadística: ** Significativa al 1 por ciento, ${ }^{* *}$ Significativa al 5 por ciento, *Significativa al 10 por ciento. No significativa a los niveles de error anteriores.

El penúltimo renglón (30) presenta el pseudo $\mathrm{R}^{2}$, también llamado de McFadden, el cual es un indicador del valor de ajuste, que no es particularmente significativo para los modelos con regresada binaria, como en este caso. Los valores de esta medida oscilan entre 0 y 1 , no obstante, valores entre 0.2 y 0.4 son altamente aceptados (Gujarati y Porter, 2010, p. 563). Por último, se muestra el valor correspondiente a la probabilidad $[\mathrm{P}(\mathrm{Yi}=1 \mid \mathrm{X})]$ de que una Mipyme se encuentre en cada una de las etapas según sea el caso. 


\section{ANÁLISIS DE LOS RESULTADOS}

Los resultados que se desprenden del modelo muestran que las Mipymes de Aguascalientes presentan una mayor probabilidad (p) de encontrarse en la tercera etapa en intensidad de uso de las TIC (0.5497). Dado el conjunto de variables explicativas, poco más de una de cada dos empresas en el estado cuenta con elementos TIC complejos que posibilitan la modificación de los procesos de gestión a través de la articulación de las áreas estratégicas hacia dentro y fuera de la organización de acuerdo con la escala de la OCDE-Cepal (2012).

En el caso contrario, de acuerdo con los resultados del modelo, se encuentra que la probabilidad de que una empresa se encuentre en la cuarta etapa de uso de las TIC es la menor, con 0.0219 , que se refiere a un uso intensivo y complejo de la tecnología, a través de la incorporación de herramientas especializadas que modifican los procesos internos y externos.

La diferencia entre la etapa 3 y 4, muestra que las Mipymes cuentan con equipo de telecomunicaciones, cómputo y software, pero presentan limitaciones para incorporar TIC especializadas que requieren de recursos humanos altamente calificados y de infraestructura tecnológica orientada a las áreas específicas de las empresas. A continuación, se analizan los resultados de los vectores de los factores internos de las empresas que inciden en la adopción de TIC en cada etapa.

\section{IV.1. Resultados del modelo}

En relación con el conjunto de las variables explicativas de los vectores de las etapas, a partir de los resultados del modelo Probit, estamos en posibilidad de mostrar cuáles son los determinantes que, en cada etapa, inciden en el uso y apropiación de las TIC en las Mipymes que fueron parte del estudio. ${ }^{2}$

\section{a) Las características de las empresas}

En relación con las condiciones de las firmas, los resultados muestran que el tamaño de la empresa es un elemento determinante en el uso de las TIC en la primera, segunda y cuarta etapa, con una significancia del 99 por ciento, pero no se observa una tendencia clara. Por otro lado, la antigüedad de la empresa no es relevante, ya que no es significativa en ningún caso.

\footnotetext{
2 La prevalencia en cada etapa no es explicada por los mismos factores, por lo que se presentan celdas con n.a (no aplica) en el cuadro 2 .
} 
El sector económico es un factor que muestra resultados mixtos. Para las empresas de la construcción, presentan una significancia negativa en las primeras etapas, y positiva en la última. En segundo lugar, se observa que las etapas 2 y 3 destacan por presentar un nivel de significancia entre el 90 y el 95 por ciento en los sectores económicos de comercio y transporte, servicios varios y educación y salud. Por último, las Mipymes dedicadas a las manufacturas y el esparcimiento, hoteles y restaurantes no presentan una tendencia significativa en ningún caso.

\section{b) Condiciones administrativas de las empresas}

Dentro de las condiciones administrativas, el nivel de escolaridad del gerente o director general se relacionan de manera positiva con la etapa en que se encuentra la empresa, por lo que, a mayores años de escolaridad, aumenta la probabilidad de que la Mipyme se encuentre en etapas superiores de uso de las TIC. Del resto de variables de la categoría, los resultados del modelo no proporcionan evidencia de algún tipo de correspondencia entre ellas y una mayor intensidad de uso.

\section{c) Equipamiento de las empresas}

Los resultados del modelo muestran que, contar con equipo de cómputo y telecomunicaciones, no es un factor determinante en la intensidad de uso en las Mipymes, aun en los casos en que se presenta equipamiento especializado (servidores), no es posible establecer una relación evidente de acuerdo con la información con que se cuenta. Un elemento diferenciador es la existencia de una red interna que conecte los equipos de cómputo entre sí, lo que permite el intercambio de información y la articulación de las actividades dentro de las Mipymes.

En relación con el tipo software utilizado por las empresas, no se observan diferencias entre el uso de programas libres (código abierto y software libre) y propietarios. En este sentido, el costo asociado a las licencias no es un factor central que refuerza los resultados que refieren que el monto del gasto no repercute en la incorporación de TIC para la producción.

\section{d) Capacidades internas}

En este último apartado, se observa que las empresas que cuentan con personal capacitado, con mayor antigüedad en el uso de computadoras, así como aquellas que cuentan con una unidad especializada en sistemas de informática, se encuentran principalmente en la cuarta etapa. Finalmente, los datos muestran que 
otorgar capacitación al interior de las firmas no es determinante para transitar hacia etapas más avanzadas en el uso de las TIC. Esto puede ser derivado del periodo de tiempo entre los cursos y el levantamiento de datos de la encuesta, ya que este es un proceso que se refleja en el mediano y largo plazo.

\section{IV.2. Factores internos de las empresas que inciden en la adopción de las TIC en las Mipymes de Aguascalientes}

Con los resultados del modelo Probit, estamos en condiciones de establecer los determinantes de intensidad de uso de las TIC en las Mypimes de Aguascalientes, que se agrupan en los cuatro vectores propuestos anteriormente (figura 2). Éstos se encuentran fundamentalmente en las capacidades internas las cuales son relevantes para que las empresas cuenten con herramientas especializadas que estandaricen los procesos y generen articulaciones hacia dentro y fuera de la organización.

Figura 2. Factores internos de las empresas que inciden en la adopción de TIC en Aguascalientes.

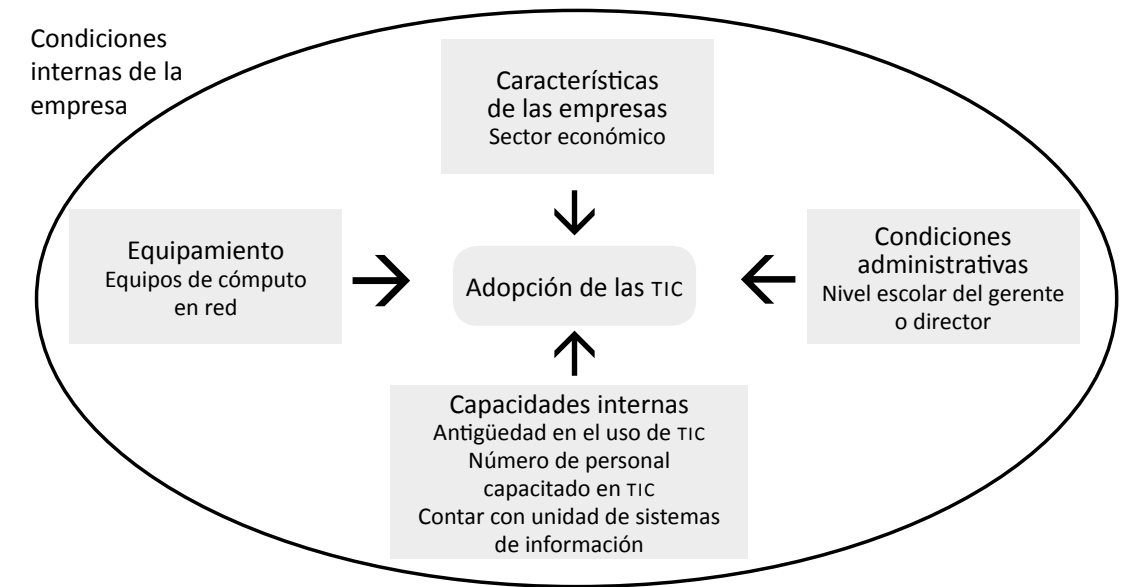

Fuente: elaboración propia.

Por medio del estudio de las Mipymes de un estado, en este trabajo se presenta un análisis que abona a la discusión sobre uso de TIC para la producción en México. A partir de los resultados del modelo, se cuenta con elementos que permiten contrastar la hipótesis en la que se basa la investigación, considerando los factores individuales presentes en cada categoría. Este enfoque micro muestra 
las diferencias de adopción de las TIC a partir de una serie de condiciones organizacionales, tecnológicas y económicas, que inciden en usos más intensivos de la tecnología dentro de los procesos de las empresas, que se reflejen en un mayor valor agregado en los productos.

De las categorías propuestas, se observa que son las capacidades internas las que presentan mayores factores que intervienen en la generación de usos más complejos dentro de las Mipymes de Aguascalientes al igual que diversos trabajos realizados a nivel internacional (Arendt, 2008; Pejic, Zorojaa and Bosilj, 2013; Bayo-Moriones y Lera-López, 2007; Bazini, Ilia y Qarri, 2011). Por lo tanto, es fundamental para el país establecer estrategias y políticas de apoyo a la formación, no sólo en las empresas, sino también a la población en general, para impulsar el manejo de las TIC y promover la incorporación de la tecnología en los diferentes ámbitos sociales y económicos (Mariscal, 2005).

En relación con el resto de los factores, se muestra que el contar con una red interna favorece que las empresas avancen en las etapas propuestas por la OCDE-Cepal, ya que el intercambio de información entre las distintas actividades permite un mayor control sobre las mismas. En relación con las condiciones administrativas, al igual que otros autores (Bayo-Moriones y Lera-López, 2007; Consoli, 2012; Arendt, 2008; Dewan y Riggins, 2005), se observa que una mayor formación del gerente o director general es un elemento central para que las empresas incorporen las TIC en sus actividades, ya que cuenta con mayor conocimiento para utilizar de manera más eficiente las inversiones en tecnología.

Por otra parte, se destaca que el tamaño de la empresa no es relevante para avanzar en las etapas propuestas por la OCDE-Cepal. Además, en el presente trabajo, no contamos con evidencia que nos permita establecer que sea un factor relevante en ninguna de las etapas. En este sentido, los resultados del modelo se encuentran en consonancia con lo presentado por Arendt en Europa (2008) y por la Cepal en América Latina (2001) con relación a que un mayor gasto no se refleja en mejor uso de la tecnología.

\section{CONCLUSIONES}

La incorporación de las TIC en las Mipymes, impulsa el crecimiento de la economía y la generación de valor. Estudios realizados en varios países muestran un efecto positivo en la producción. En el caso particular de Aguascalientes, Maldonado, Martínez García, Aguilera, y González en el 2010 habían mostrado la relación positiva entre las TIC y el rendimiento de las Pymes, por lo que el objetivo del presente fue abonar a esta discusión, estableciendo los determinantes internos que inciden en la incorporación de las TIC en las Mipymes, a partir del 
análisis de los resultados obtenidos del modelo Probit aplicado a los datos de una encuesta realizada a las Mipymes de Aguascalientes.

La evidencia sugiere que la cantidad de recursos monetarios dedicada a la adquisición y mantenimiento de las TIC no es condición suficiente por sí misma, para avanzar hacia etapas más complejas de uso. Por lo que debe estar acompañada de una estrategia de corto, mediano y largo plazo, destinada a estimular la capacitación de los trabajadores en el uso de herramientas especializadas para las actividades de la empresa.

El modelo muestra que hay algunos sectores que son menos propensos a incorporar las TIC en los procesos internos en particular, la manufactura y el esparcimiento, hoteles y restaurantes, por lo que es necesario establecer estrategias de apoyo para impulsar su adopción en las Mipymes del estado.

Asimismo, un mayor conocimiento de los directivos en relación con el valor que incorpora las TIC dentro de los procesos es un factor para impulsar usos más complejos y eficientes del gasto en tecnología, a través de la adquisición de equipamiento y software que responda a las necesidades de las empresas.

Este trabajo es una propuesta para abordar el análisis de un tema multifactorial como lo es la brecha digital de segundo orden en las empresas mexicanas, debido a la falta de información en el país en relación con los factores internos de las Mipymes que intervienen en usos más complejos de las TIC. Es necesario considerar que se realizó con información de un estado altamente industrializado y conectado, por lo que no se pueden extrapolar los resultados a otras regiones, pero es un elemento de aporte para futuras investigaciones en el tema.

\section{REFERENCIAS BIBLIOGRÁFICAS}

Arendt, Lukasz (2008), "Barriers to ict Adoption in smes: How to Bridge the Digital Divide?", Journal of Systems and Information Technology, 10 (2), pp. 93-108.

Bazini, Evelina; Ilia, Djalna y Qarri, Albert (2011), "Barriers of ICT Implementations Within SMEs in Service Sector in Albania", EuroEconomica, 3 (29), pp. 114-120.

Bayo-Moriones, Alberto y Lera-López, Fernando (2007), "A Firm-Level Analysis of Determinants of ICT Adoption in Spain”, Technovation, 27 (6), PP. 352-366.

Billón-Currás, Margarita; Lera-López, Fernando y Ortiz-Serrano, Salvador (2007), "Evidencias del impacto de las TIC en la productividad de la empresa. ¿Fin de la paradoja de la productividad?", Cuadernos de Economía, 30 (82), pp. 5-36.

Casalet, Mónica; González, Leonel; Buenrostro, Edgar; Oliver, Rubén y Becerril, Gabriela (2008), El impacto de las políticas e instituciones locales y sectoriales en el desarrollo de "clusters" en México: el caso del sector de software, Organización Internacional del Trabajo, México, 115 pp. 
Comisión Económica para América Latina (Cepal) (2013), Economía digital para el cambio estructural y la igualdad, Santiago, $130 \mathrm{pp}$.

Comisión Económica para América Latina-Unión Europea (2010), Las TIC para el crecimiento y la igualdad: renovando las estrategias de la sociedad de la información, Santiago, 113 pp.

Consoli, Domenico (2012), "Literature Analysis on Determinants Factors and the Impact of ICT in SMEs", Procedia-Social and Behavioral Sciences, 62 (octubre), pp. 93-97.

Cruz-Jesus, Federico; Oliveira, Tiago y Bacao, Fernando (2012), "Digital Divide Across the European Union”, Information \& Management, 49 (6), pp. 278-291.

Davis, Fred (1989), "Perceived Usefulness, Perceived Ease of Use, and User Acceptance of Information Technology", MIS Quarterly, 13 (3), pp. 319-340.

Dewan, Sanjeev y Riggins, Frederick (2005), “The Digital Divide: Current and Future Research Directions", Journal of the Association for Information Systems, 6 (12), pp. 298-337.

Diario Oficial de la Federación (DOF) (2009), "Acuerdo por el que se establece la estratificación de las micro, pequeñas y medianas empresas", 30 de junio, disponible en http://dof.gob.mx/nota_detalle.php? codigo $=5096849 \&$ fecha $=30 / 06 / 2009$, consultado el 12 de mayo de 2018.

Fabiani, Silvia; Schivaldi, Faviano y Trento, Sandro (2005), "ICT Adoption in Italian Manufacturing: Firm-level Evidence", Industrial and Corporate Change, 14 (2), pp. 225-249.

Forman, Chris y Goldfarb, Avi (2005), "Diffusion of Information and Communication Technologies to Businesses", working paper, pp. 1-69.

Galliano, Danielle y Roux, Pascale (2008), "Organizational Motives and Spatial Effects in Internet Adoption and Intensity of Use: Evidence from French industrial Firms", Annals of Regional Science, 42 (2), pp. 425-448.

Galve-Gorriz, Carmen y Gargallo-Castel, Ana (2004), "Impacto de las tecnologías de la información en la productividad de las empresas españolas", documento de trabajo 2004-05, pp. 1-26.

García, Domingo y Martínez-Serna, María (2009), Innovación y cultura empresarial de las Mipyme (Micro, pequeña y mediana empresa) Estado de Aguascalientes, Universidad Autónoma de Aguascalientes y Universidad Politécnica de Cartagena, México, $181 \mathrm{pp}$.

Gefen, David y Straub, Detmar (2000), “The Relative Importance of Perceived Ease of Use in is Adoption: A Study of E-Commerce Adoption", Journal of the Association for Information Systems, 1 (8), pp. 1-28

Greene, William (1998), Análisis econométrico, $3^{\mathrm{a}}$ ed., Prentice Hall, España, 914 pp. Gujarati, Damodar y Porter, Dawn, (2010), Econometría, 3a ed., Mc Graw Hill, México, $944 \mathrm{pp}$. 
Hawash, Ronia y Lang, Guenter (2010), "The Impact of Information Technology on Productivity in Developing Countries", working paper series \# 19, German University in Cairo, pp. 1-22.

Hollenstein, Heinz (2004), "Determinants of the Adoption of Information and Communication Technologies (ICT). An empirical Analysis Based on Firm-level Data for the Swiss Business Sector", Structural Change and Economic Dynamics, 15 (3), pp. 315-342.

Ifinedo, Princely (2012), E-Business-Applications and Global Acceptance, INTECH, Nova Scotia, $146 \mathrm{pp}$.

Instituto Nacional de Estadística y Geografía (Inegi) (2015), Banco de Información Económica, Disponible en http: //www.Inegi.org.mx/sistemas/bie, consultado el 22 de abril de 2016.

Kotelnikov, Vadim (2007), Small and Medium Enterprises and ict, Asia-Pacific Development Information Programe. E-Primers for the Information Economy, Society and Polity, Tailandia, United Nations, Tailandia, $27 \mathrm{pp}$.

Maldonado, Gonzalo; Martínez, María; García, Domingo; Aguilera, Luis y González, Martha (2010), "La influencia de las TICs en el rendimiento de la Pyme de Aguascalientes", Investigación y Ciencia de la Universidad Autónoma de Aguascalientes, 47, pp. 57-65.

Martínez, María; García, Domingo y Maldonado, Gonzalo (2010), Innovación y gestión del conocimiento en las Pymes de Aguascalientes, Universidad Autónoma de Aguascalientes, Aguascalientes, $232 \mathrm{pp}$.

Mariscal, Judith (2005), "Digital Divide in a Developing Country", Telecommunication Policy, 29, pp. 409-428.

Matthews, Paul (2007), "ICT Assimilation and sme expansion", Journal of International Development, 19 (86), pp. 817-827.

Molina, Mariela; Rotondo, Sebastian y Yoguel, Gabriel (2013), "El impacto de las TIC en la productividad del trabajo: algunos indicios para las Pymes del sector manufacturero argentino", en Novick, Marta y Rotondo, Sebastian, El desafío de las TIC en Argentina. Crear capacidades para la generación de empleo, Cepal, Santiago, pp. 107-136, 290 pp.

Novick, Marta; Rotondo, Sebastian y Yoguel, Gabriel (2013), "Cambio estructural, conductas tecnológicas y empleo. El tránsito hacia un estudio más complejo de las TIC en la Argentina”, en Novick, Marta y Rotondo, Sebastian, El desafío de las тIC en Argentina. Crear capacidades para la generación de empleo, Cepal, Santiago, pp. 17-42, 290 pp.

Organización para la Cooperación y el Desarrollo Económicos (OCDE)-Comisión Económica para América Latina, (Cepal) (2012), Perspectivas económicas de América Latina 2013. Políticas de Pymes para el cambio estructural, Cepal, Santiago, 189 pp. 
Pejic Bacha, Mirjana; Zorojaa, Jovana y Bosilj, Vesna (2013), "Determinants of Firms Digital Divide: A Review of Recent Research”, Procedia Technology, 9, pp. 120-128.

Pick, James y Nishida, Tetsishi (2015), "Digital Divides in the World and its Regions: A Spatial and Multivariate Analysis of Technological Utilization", Technological Forecasting \& Social Change, 91 (February), pp. 1-17.

Rivas, Diego y Stumpo, Giovanni (2013), "Las TIC en el tejido productivo de América Latina”, en Novick, Marta y Rotondo, Sebastian, El desafío de las TIC en Argentina. Crear capacidades para la generación de empleo, Cepal, Santiago, pp. 43-78, 290 pp.

Rovira, Sebastián; Santoleri, Pietro y Stumpo, Giovanni (2013), “Incorporación de TIC en el sector productivo: uso y desuso de las políticas públicas para favorecer su difusión", en Rovira, Sebastián y Stumpo Giovanni, Entre mitos y realidades. TIC, políticas públicas y desarrollo productivo en América Latina, Cepal, Santiago, pp. 17-54, Santiago, 340 pp.

Santinha, Goncalo y Soares, Antonio (2004), "SMEs and ICTs adoption: a new challenge to regional policies", working paper, Department of Environmental Studies and Planning, University of Aveiro, pp. 1-14.

Schatan, Claudia y Enríquez, Leobardo (2014), "El sector de tecnologías de la información y la comunicación y las políticas industriales: un tema actual para México", Proceeding of the 8 CPR LATAM Conference, pp. 1-35.

Tello-Leal, Edgar (2008), "Las tecnologías de la información y comunicación (TIC) y la brecha digital: su impacto en la sociedad de México", Revista Universidad y Sociedad del Conocimiento, 4 (2), pp. 1-8.

Tello-Leal, Edgar; Sosa-Reyna, Claudia y Tello-Leal, Diego (2012), “The Digital Divide: ict Development Indices in Mexico", Journal of Community Positive Practices, 4, pp. 797-812.

Ueki, Yasushi; Tsuji, Masatsugu y Cárcamo, Rodrigo (2005), Tecnología de la información y las comunicaciones (TIC) para el fomento de las Pymes exportadoras en América Latina y Asia oriental, Cepal, Santiago, 136 pp.

Wielicki, Tom y Arendt, Lukasz (2010), "A Knowledge-driven Shift in Perception of ict Implementation Barriers: Comparative Study of US and European SMEs", Journal of Information Science, 36 (162), pp. 162-174.

Wielicki, Tom y Cavalcanti, Gustavo (2006), "Study of Digital Divide: Measuring ICT Utilization and Implementation Barriers Among SMEs of Central California", en Abramowicz, W. y Mayr, H. (eds.), 9th International Conference on Business Information Systems, pp. 277-294. 
ANEXO

\section{Actividades de las etapas de apropiación de las TIC en las Mipymes de Aguascalientes.}

Etapa Descripción Indicadores considerados en el cuestionario

No cuenta con computadoras, acceso a internet, ni telefonía.

Equipamiento básico de TIC:

Cuenta con computadoras

Cuenta con acceso a internet

Cuenta con acceso a las TIc básicas

Etapa 2 (computadoras e internet) cuyos usos se concentran en usos rutinarios de producción y administración.
Usos de internet básicos:

Correo electrónico

Consulta de información general

Compras por internet

Operaciones bancarias

Pago de servicios gubernamentales

Reclutamiento y selección de personal

Monitoreo del mercado

Telefonía VoiP

Usos de las TIC en las actividades de la empresa:

Facturación

Contabilidad y nómina

Distribución y ventas

Compras y pagos a proveedores

Control y manejo de inventario

Aplicaciones de fabricación y diseño de pro-

ductos (no en los servicios)

Control de procesos de calidad (no en los servicios)

Usos de Internet asociados a la producción:

Ventas por internet

Atención a proveedores

Atención a clientes

Capacitación y entrenamiento de personal

Aplicaciones en la nube

Cuenta con página de internet

Programas de gestión empresarial:

Hace uso de CRM

Hace uso de BI

Hace uso de ERP

Hace uso de BPM

Hacen uso de CMS

Equipamiento especializado:

Cuenta con unidad de $\mathrm{TI}$

Cuentan con intranet

Fuente: elaboración propia con base en oCDE-Cepal, 2012 y Kotelnikov, 2007. 